\title{
3 Research Square

\section{The Use And Benefits of 3D Digital Microscope In Hepatic Artery Reconstruction During Living Donor Liver Transplant}

\section{Ching-Min Lin}

Changhua Christian Hospital

\section{Shih-Lung Lin}

Changhua Christian Hospital

\section{Yu-Ju Hung}

Changhua Christian Hospital

\section{Chia-En Hsieh}

Changhua Christian Hospital

\section{Yao-Li Chen}

Changhua Christian Hospital

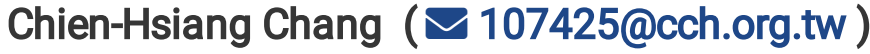

Changhua Christian Hospital

\section{Research Article}

Keywords: 3D digital microscope, exoscope, exoscopic anastomosis, artery anastomosis

Posted Date: September 16th, 2021

DOI: https://doi.org/10.21203/rs.3.rs-865452/v1

License: (1) This work is licensed under a Creative Commons Attribution 4.0 International License. Read Full License 


\section{Abstract}

Background: To compare the intraoperative process of hepatic artery anastomosis using conventional microscope and novel 3D digital microscope and to share our institute's experience for better surgical training.

Method: A retrospective cohort study with 46 hepatic artery reconstructions in living donor liver transplant patients via operational microscope (Control group) and 3D digital microscope Mitaka Kestrel View II (Study group). We then discuss and share our institution's experience to improve surgical training.

Results: Both operation instruments provide effective and comparable results. There was no statistical significance regarding operational objective results between conventional microscope and exoscope. Both instruments have no hepatic artery size limit, and both have complete vessel patency rate.

Conclusions: There was no statistical differences regarding hepatic artery anastomosis between microscope and exoscope cohorts. However, exoscope provided better ergonomics in the operation room and lessen musculoskeletal strain, allowing surgeons to work in a more neutral and comfortable posture and allow first assistant to learn and assist more effectively. Using exoscope with micro-forceps and modified tie technique make artery reconstruction easier.

\section{Background}

Living donor liver transplant (LDLT) is considered the best treatment option for advanced liver disease. The most crucial and complex step in LDLT is the reconstruction of hepatic artery and risk of hepatic artery-related complications. Early experience with LDLT by using surgical loupe resulted in high rates of hepatic artery thrombosis, hepatic necrosis, graft loss and mortality up to $50 \%{ }^{1,2}$ Patients who develop hepatic artery thrombosis have decreased survival even if the undergo re-transplantation. ${ }^{3}$ Since the introduction of microvascular anastomosis, the reported incidence of hepatic artery reconstruction complication rates was dramatically reduced to between 0 and $6 \% .{ }^{4}$ Hence, a shift to microscopic anastomosis was made. However, from surgical loupe to microscope, due to the difference in the entire surgical equipment and technology, the learning curve becomes very long. Microvascular reconstruction is the trend in most hospitals, so here we share our experience with 3D digital microscopy. It is a novel high-definition digital camera, the conversion of digital data to allow us to have stereovision and since it does not rely on the eyepiece, surgeons can have larger range of motion and the operation method is similar to that of conventional microscope so the learning curve after switching from conventional microscopy to exoscope is shorten. This retrospective study compared a single microsurgeon's intraoperative process on hepatic artery anastomosis during living donor liver transplant using operational microscopy versus using 3D digital microscopy and discuss the benefit of newly emerging exoscopic technology.

\section{Methods}


A retrospective cohort study of hepatic artery reconstruction as part of living donor liver transplant patients between 2018 April and 2019 April done by single plastics surgeon Dr. Shih-Lung Lin at medical center Changhua Christian Hospital was conducted. A total of 46 charts were examined: 23 patients received microsurgery via operational microscopy (Control group) and 23 patients received microsurgery via 3D digital microscopy Mitaka Kestrel View II (Study group). Collected data include patient and donor's demographic data, medical history, tissue ischemia time and procedural characteristics. Surgical outcome as hepatic artery perfusion was evaluated via Doppler ultrasound evaluated immediately post anastomosis and before reversal of general anesthesia at the end the operation.

\section{Results}

The study included 46 patients who underwent hepatic artery reconstruction as part of living donor liver transplant. Demographic and intraoperative features are as Table 1. Overall, gender was distributed equally between control and study group, each group having 16 males in 23 patients. There was no statistical significance between recipient age and donor age in both groups. Both control and study group have 2 patients (8.7\%) with hypertension. Control group has 8 patients $(34.8 \%)$ with diabetes mellitus and 12 smokers (52.2\%), whereas study group have 5 patients (21.7\%) with diabetes mellitus and 15 smokers (65.2\%). These demographic data did not provide no clinical significance. In the control group, the recipient artery size averaged $3.65 \pm 0.647 \mathrm{~mm}$ and donor artery size averaged $2.43 \pm 0.662 \mathrm{~mm}$. In the study group, the recipient artery size averaged $3.91 \pm 0.900 \mathrm{~mm}$ and donor artery size averaged $2.70 \pm$ $0.765 \mathrm{~mm}$. Intraoperative variables including average cold ischemia time, warm ischemia time, anastomosis time, and blood loss were not at all significant difference in favorable of hepatic artery anastomosis between the two comparative cohorts. 
Table 1

Demographic and intraoperative features between conventional microscope (control group) and exoscopy (study group)

\begin{tabular}{|c|c|c|c|c|}
\hline \multirow{2}{*}{$\begin{array}{l}\text { Demographic and clinical } \\
\text { features }\end{array}$} & \multirow{2}{*}{$\begin{array}{l}\text { Control group } \\
n=23\end{array}$} & \multicolumn{2}{|l|}{ Study group } & \multirow{2}{*}{$\begin{array}{l}\text { Total } \\
n=46\end{array}$} \\
\hline & & $\mathrm{n}=\mathbf{2 3}$ & $p$ & \\
\hline & Mean \pm SD & Mean \pm SD & & Mean \pm SD \\
\hline & (range) & (range) & & (range) \\
\hline \multirow[t]{2}{*}{ Recipient age (years) } & $54.78 \pm 7.292$ & $53.78 \pm 8.339$ & \multirow[t]{2}{*}{0.575} & $54.28 \pm 7.762$ \\
\hline & $(35-67)$ & $(39-70)$ & & $(35-70)$ \\
\hline \multirow[t]{2}{*}{ Donor age (years) } & $32.30 \pm 9.152$ & $32.22 \pm 11.342$ & \multirow[t]{2}{*}{0.575} & $18.0 \pm 54.0$ \\
\hline & $(19-54)$ & $(18-52)$ & & $(32.26-10.190)$ \\
\hline \multirow[t]{2}{*}{ Recipient artery size (mm) } & $3.65 \pm 0.647$ & $3.91 \pm 0.900$ & \multirow[t]{2}{*}{0.204} & $2.78 \pm 0.786$ \\
\hline & $(2.0-5.0)$ & $(2.0-6.0)$ & & $(2.0-6.0)$ \\
\hline \multirow[t]{2}{*}{ Donor artery size $(\mathrm{mm})$} & $2.43 \pm 0.662$ & $2.70 \pm 0.765$ & \multirow[t]{2}{*}{0.216} & $2.57 \pm 0.720$ \\
\hline & $(2.00-4.00)$ & $(2.0-4.0)$ & & $(2.0-4.0)$ \\
\hline \multirow[t]{2}{*}{ Anastomosis time $(\mathrm{min})$} & $25.22 \pm 7.373$ & $23.91 \pm 5.316$ & \multirow[t]{2}{*}{0.422} & $24.57 \pm 6.390$ \\
\hline & $(12.0-38.0)$ & $(15.0-35.0)$ & & $(12.0-38.0)$ \\
\hline \multirow[t]{2}{*}{ Blood loss (ml) } & $\begin{array}{l}2985.65 \pm \\
2021.583\end{array}$ & $\begin{array}{l}4854.35 \pm \\
7992.623\end{array}$ & \multirow[t]{2}{*}{0.391} & $\begin{array}{l}3875.00 \pm \\
5848.901\end{array}$ \\
\hline & $(800.0-1000.00)$ & $(500.0-40000.0)$ & & $(500.0-40000.0)$ \\
\hline \multirow[t]{2}{*}{ Cold ischemia time (min) } & $42.00 \pm 22.817$ & $49.70 \pm 21.231$ & \multirow[t]{2}{*}{0.059} & $45.85 \pm 22.137$ \\
\hline & $(13.0-128.0)$ & $(25.0-130.0)$ & & $(13.0-130.0)$ \\
\hline \multirow[t]{3}{*}{ Warm ischemia time (min) } & $19.83 \pm 5.589$ & $21.35 \pm 6.050$ & \multirow[t]{2}{*}{0.208} & $20.59 \pm 5.810$ \\
\hline & $(13.0-33.0)$ & $(13.0-35.0)$ & & $(13.0-35.0)$ \\
\hline & $(\%)$ & $(\%)$ & & $(\%)$ \\
\hline Gender (male) & 16(69.6) & 16(69.6) & 1.000 & (32)69.6 \\
\hline Diabetes mellitus & $8(34.8)$ & $5(21.7)$ & 0.326 & 13(28.3) \\
\hline Hypertension & $2(8.7)$ & $2(8.7)$ & 1.000 & $4(8.7)$ \\
\hline Smoking & $12(52.2)$ & $15(65.2)$ & 0.369 & $27(58.7)$ \\
\hline
\end{tabular}


This single center single surgeon experience retrospective cohort study investigates the usage and benefits of 3D digital microscope in hepatic artery reconstruction during living donor liver transplant. Although operative techniques for hepatic transplant have matured enough to yield high success rates, we propose a few modifications that can be adopted to make vascular anastomosis in hepatic transplant easier for young surgeons.

\section{The Use of Micro-forceps}

Forceps are commonly used during operations and each surgical speciality has its own custom forceps that is designed to suit their operative approaches (Figure 1). The $15 \mathrm{~cm}$ long forceps is a standard instrument in most general surgery operations, yet when used in hepatic artery anastomosis, it is not effective. As seen in Figure 2, the end of a $15 \mathrm{~cm}$ long forceps, when held in the hand of surgeon during artery anastomosis, is against the operator's palm and the operator's fingers are pinched to have effective grip, which is tiresome for the hand and provides little lever working space. Furthermore, the surgeon's hand motion is often limited by rib encasing the liver. To overcome this limitation, we use a $25 \mathrm{~cm}$, or $20 \mathrm{~cm}$, long micro-forceps for the procedure (Figure 1, right). As seen in the Figure 2, the extended length of this micro-forceps allows surgeons' hand to work in a more relaxed grip with a working field that is wider and deeper. Leverage in longer forceps allow precise movement at forceps' tip without much hand movement, which is essential for artery reconstruction. When both the primary surgeon and assistant surgeon use micro-forceps during the procedure (Figure 2, bottom), the assistant is able to provide assistance more precisely without blocking the surgeon's working field and the anastomosis can proceeded smoothly and efficiently.

\section{Tying Surgical Knots}

Surgical residents were conventionally taught to hold the needle-holder with their dominant hand, usually the right-hand, and have the forceps in their non-dominant hand, usually the left-hand. Surgical knots were made by circling the forceps around the needle holder. However, during hepatic artery anastomosis, the rib encasing the liver will limit the surgeon's field of exercise and since the since the working field is vertically beneath the hand, as seen in figure, surgical knots are often loose due to inadequate tie. Hence, we propose circling the needle-holder around the still forceps. The right-hand held needle-holder has more working space than the left-hand forceps, this allows more effective and firmer surgical knots to be made than the conventional method.

\section{The Operation Setup: Operative microscope}

Figure 3 shows the operation setup with conventional operative microscope (Control group). The main operator is on the left side and assistant operator on the right side of the microscope. To accommodate the microscope, operators often maintain a forward leaning, stiff neck and shrugged shoulder position. If the arm-board is used in some operations, operators may need to stand with twisted trunk position and slanted shoulder to gain visualization to the microscopic field. In addition, with only one objective lens 
and the microscope is usually set up to suit the primary surgeon, assistant surgeon often stands in awkward non-ergonomic positions.

\section{The Operation Setup: Exoscope}

Figure 4 shows the operative setup with the use of 3D digital microscope Mitaka Kestrel View II (Study group). As seen in Figure 5, both operators are standing in upright position with relaxed shoulder. A real time projection of the operation field that allows all bystanders, such as scrub nurse, anesthesiologist, surgical residents, to learn intraoperative techniques and follow the progress of the operation. In addition, looking at a projection screen is easier on the eyes for surgeons with presbyopia. Less physical discomfort with heighten surgeons' concentration and willingness to teach and learn throughout this complicated surgery.

\section{Benefits of Our Approach}

Surgeons endure long and strenuous training years with high level of stress to perfect their surgical techniques to improve patients' surgical outcome and prognosis. This pursuit of perfection comes at the cost of surgeons physical and mental health. Studies have found that $60-90 \%$ of all surgeons experience painful musculoskeletal conditions in their neck, back or shoulders. ${ }^{5,6,7}$ Microsurgeons experience these symptoms during or after microscope use. ${ }^{8}$ Our approach with using micro-forceps instead of standard forceps stabilizes the hand during vascular reconstruction with more precise movement and the modified suture tying method provides larger working field with firmer ties made. The use of exoscopes, such as 3D microscopy, can neutralize mal-posture and alleviate work-related injuries. However, surgeons cannot benefit themselves at the cost of jeopardizing patient's health. Although Pafitanis et al. found that exoscopic microvascular anastomosis was more time consuming, our comparative analysis of hepatic artery reconstruction done via operational microscopy and via 3D digital microscopy showed no statistical nor clinical significance in operative outcome (Table 1). Both instruments have no hepatic artery size limit, and both have complete vessel patency rate. This non-inferiority is in concordance to previous study. ${ }^{8}$

Rosenblatt et al. analyze surgeons' intraoperative posture and found three most common malposition that contributed musculoskeletal injuries: forward head position, improper should elevation and internal rotations, and pelvic girdle asymmetry. As in Figure 3, the forward leaning position, stiff neck position and shrugged shoulder causes fatigue in deltoid and trapezius muscles. Due to obstacles, surgeons need to maintain a twisted position to gain visualization of the operation field. This sustained twisting causes asymmetrical loading on back and leg muscles, leading to work-related musculoskeletal pain. Other risk factors that led to increased muscle activity and muscle fatigue include prolong static posture, hyperflexion of cervical spine and back-bent posture. ${ }^{6,11,12}$ Hence operating with a more neutral position should be reminded and reinforced among surgeons.

Modern digital exoscopes have its forte with surgeons. Its application ranges from open surgery to microsurgery and its ergonomics design, the projection of microscopic image onto a 3D monitor, allow 
surgeons to operate in a heads-up position, alleviating cervical musculoskeletal fatigue and work-related injuries. For surgeons with presbyopia, looking at a distant screen is more comfortable than a close-up image. Moreover, this real-time projection allows all active participants in the operation room to see the progress of the operation and allow bystanding surgical residents to learn intraoperative skills. 3D digital microscopy is an asset to surgeons in non-reconstructive specialties because of its similar operative method with laparoscopy and the switch from microscope to exoscope with good quality outcome can be achieved in relatively short practice time. ${ }^{13}$

\section{Conclusion}

Vascular anastomosis is the foundation of plastics and reconstructive surgery and technological advancements in this field have revolutionized the approach to reconstructive microsurgery. 3D microscopy is a novel exoscope that is at least equivalent to operating microscopy in intraoperative procedure. When microvascular anastomosis is performed by experts using 3D microscopy, there is no negative clinical impact to patients and offers improved ergonomics, image quality and accessibility to the surgical field. New technologies do have their forte and as surgical residents learn to use these instruments and techniques, comparative analysis of surgical outcome for patient is just as important as operator's overall comfort during operation.

\section{Declarations}

All methods in this study were conducted in accordance with the Declaration of Helsinki and approved by the institutional review board and ethics committee of Changhua Christian Hospital (CCH IRB No. 181020), which waived the need for patient consent.

Ethics approval and consent to participate: The study was conducted in accordance with the Declaration of Helsinki and approved by the institutional review board and ethics committee of Changhua Christian Hospital (CCH IRB No. 181020), which waived the need for patient consent.

Consent for publication: Not applicable.

Availability of data and materials: The datasets used and/or analyzed during the current study are available from the corresponding author on reasonable request.

Competing interests: The authors declare that they have no competing interests.

Funding: This study did not receive any specific grant from funding agencies in the public, commercial or not-for-profit sectors.

Authors' contributions: CML and SHL contributed to writing of the manuscript. SHL, YJH and YLC participated in performing the transplant surgery. CEH was in charge of data collection and analysis. YLC was in charge of research design. 
Acknowledgements: We would like to thank our patients for entrusting us and to all medical personnel for their unconditional care to our patients even when enduring musculoskeletal and mental fatigue during the long working hours.

\section{References}

1. Bodor R, Greenberg JI, Abrams RA, et al. Microvascular hepatic artery reconstruction in pediatric abdominal transplantation: pearls and pitfalls. Pediatr Transplant. 2007;11(5):471-476. doi:10.1111/j.1399-3046.2007.00740.x

2. Broelsch CE, Burdelski M, Rogiers X, et al. Living donor for liver transplant. Hepatology 1994;20:49S$55 S$.

3. Vrochides $D$, Hassanain $M$, Metrakos $P$, et al. Re-vascularization may not increase graft survival after hepatic artery thrombosis in liver transplant recipients. Hippokratia. 2010;14(2):115-118.

4. Mori K, Nagata I, Yamagata S, et al. The introduction of microvascular surgery to hepatic artery reconstruction in living-donor liver transplantation-its surgical advantages compared with conventional procedures. Transplantation. 1992;54(2):263-268. doi:10.1097/00007890-19920800000014

5. Cavanagh J, Brake M, Kearns D, Hong P. Work environment discomfort and injury: an ergonomic survey study of the American Society of Pediatric Otolaryngology members. Am J Otolaryngol. 2012;33(4):441-446. doi:10.1016/j.amjoto.2011.10.022

6. Esposito C, El Ghoneimi A, Yamataka A, et al. Work-related upper limb musculoskeletal disorders in paediatric laparoscopic surgery. A multicenter survey. J Pediatr Surg. 2013;48(8):1750-1756. doi:10.1016/j.jpedsurg.2013.01.054

7. Liang CA, Levine VJ, Dusza SW, Hale EK, Nehal KS. Musculoskeletal disorders and ergonomics in dermatologic surgery: a survey of Mohs surgeons in 2010. Dermatol Surg. 2012;38(2):240-248. doi:10.1111/j.1524-4725.2011.02237.x

8. Soueid A, Oudit D, Thiagarajah S, Laitung G. The pain of surgery: pain experienced by surgeons while operating. Int J Surg. 2010;8(2):118-120. doi:10.1016/j.ijsu.2009.11.008

9. Pafitanis G, Hadjiandreou M, Alamri A, Uff C, Walsh D, Myers S. The Exoscope versus operating microscope in microvascular surgery: A simulation non-inferiority trial. Arch Plast Surg. 2020;47(3):242-249. doi:10.5999/aps.2019.01473

10. Rosenblatt PL, McKinney J, Adams SR. Ergonomics in the operating room: protecting the surgeon. $J$ Minim Invasive Gynecol. 2013;20(6):744. doi:10.1016/j.jmig.2013.07.006

11. Szeto GP, Cheng SW, Poon JT, Ting AC, Tsang RC, Ho P. Surgeons' static posture and movement repetitions in open and laparoscopic surgery. J Surg Res. 2012;172(1):e19-e31. doi:10.1016/j.jss.2011.08.004

12. Berguer R, Rab GT, Abu-Ghaida H, Alarcon A, Chung J. A comparison of surgeons' posture during laparoscopic and open surgical procedures. Surg Endosc. 1997;11(2):139-142. 
doi:10.1007/s004649900316

13. Hafez A, Elsharkawy A, Schwartz C, et al. Comparison of Conventional Microscopic and Exoscopic Experimental Bypass Anastomosis: A Technical Analysis. World Neurosurg. 2020;135:e293-e299. doi:10.1016/j.wneu.2019.11.154

\section{Figures}
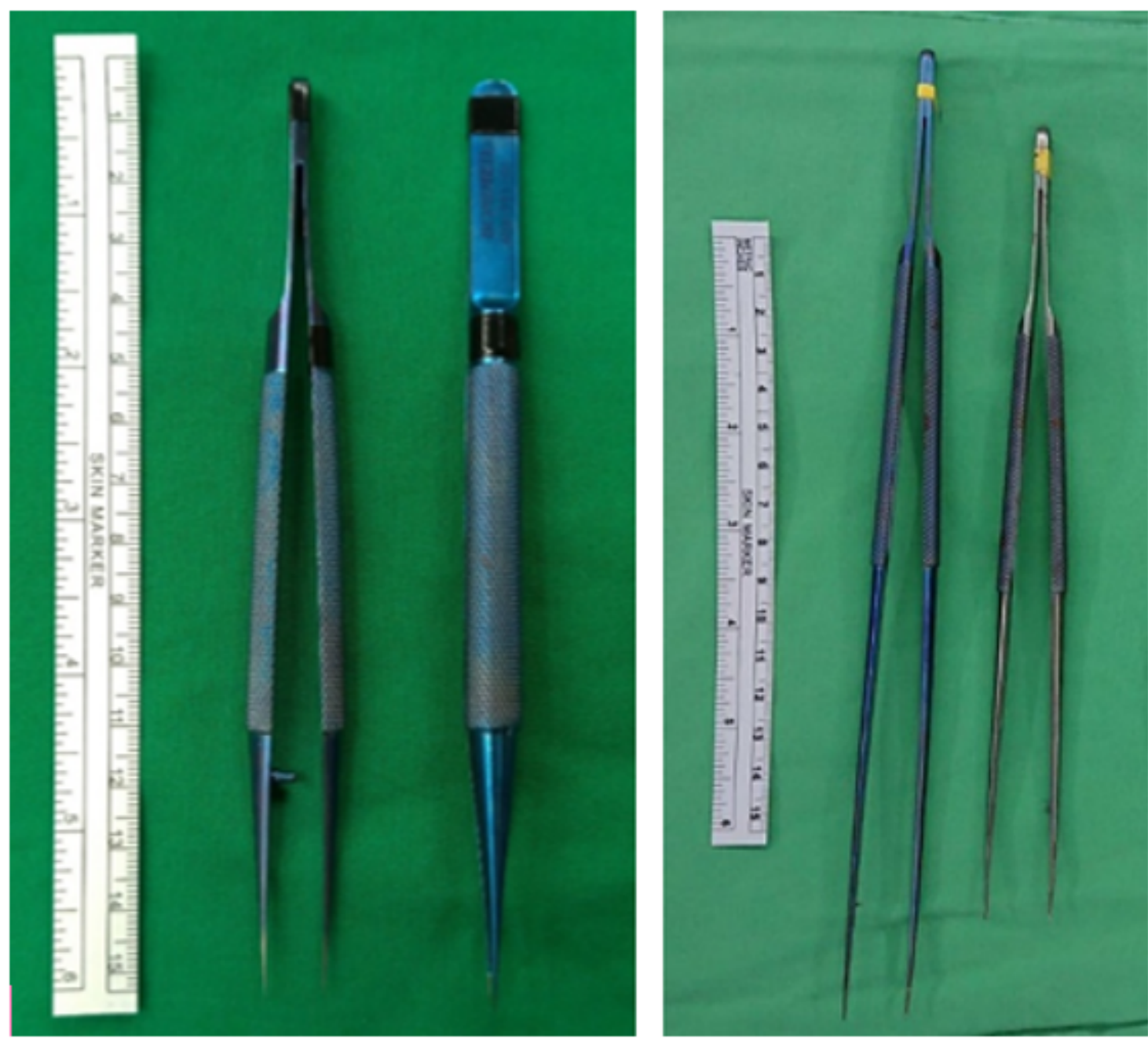

\section{Figure 1}

Forceps and micro-forceps. (Left) A $15 \mathrm{~cm}$ long tissue forceps, a standard instrument in most general operations. (Right) A $25 \mathrm{~cm}$ long and a $20 \mathrm{~cm}$ long micro-forceps, used during hepatic artery anastomosis at our institution. 

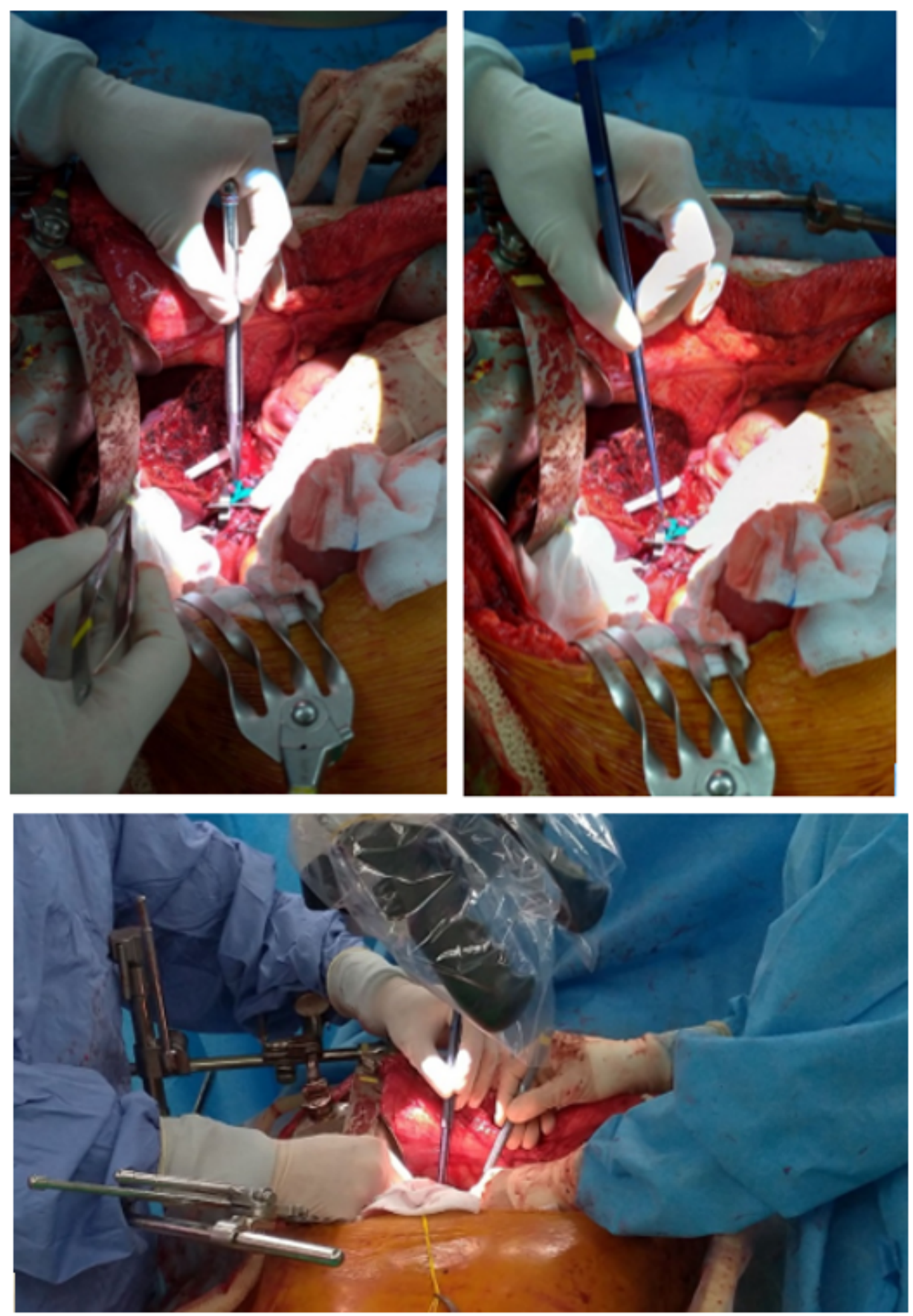

\section{Figure 2}

Working with forceps and micro-forceps. (Left) Surgeon using the standard $15 \mathrm{~cm}$ long forceps. (Right) Surgeon using the $25 \mathrm{~cm}$ long micro-forceps. (Bottom) Surgeon and first assistant both using microforceps. 

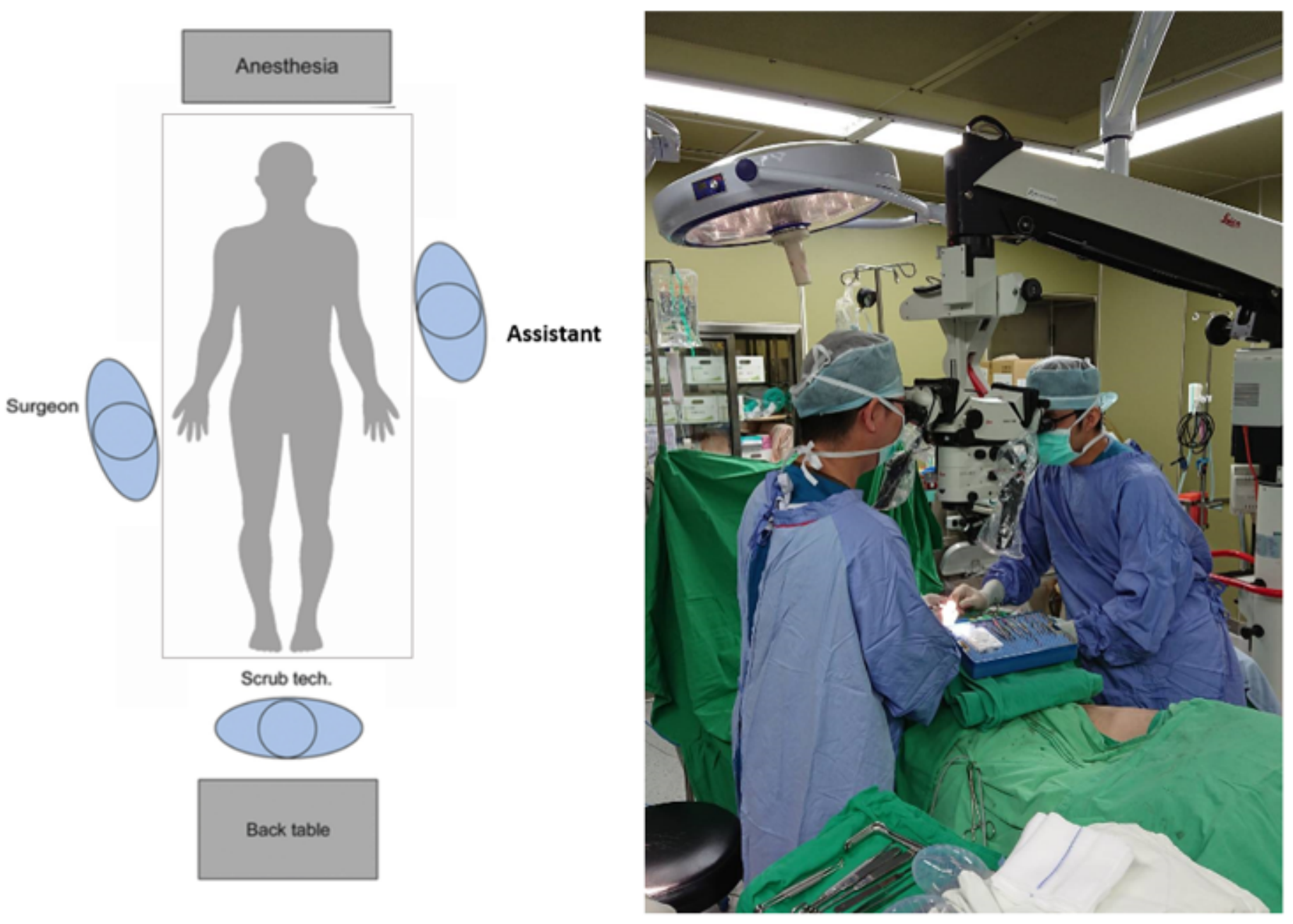

\section{Figure 3}

Operative setup for conventional microscope. 


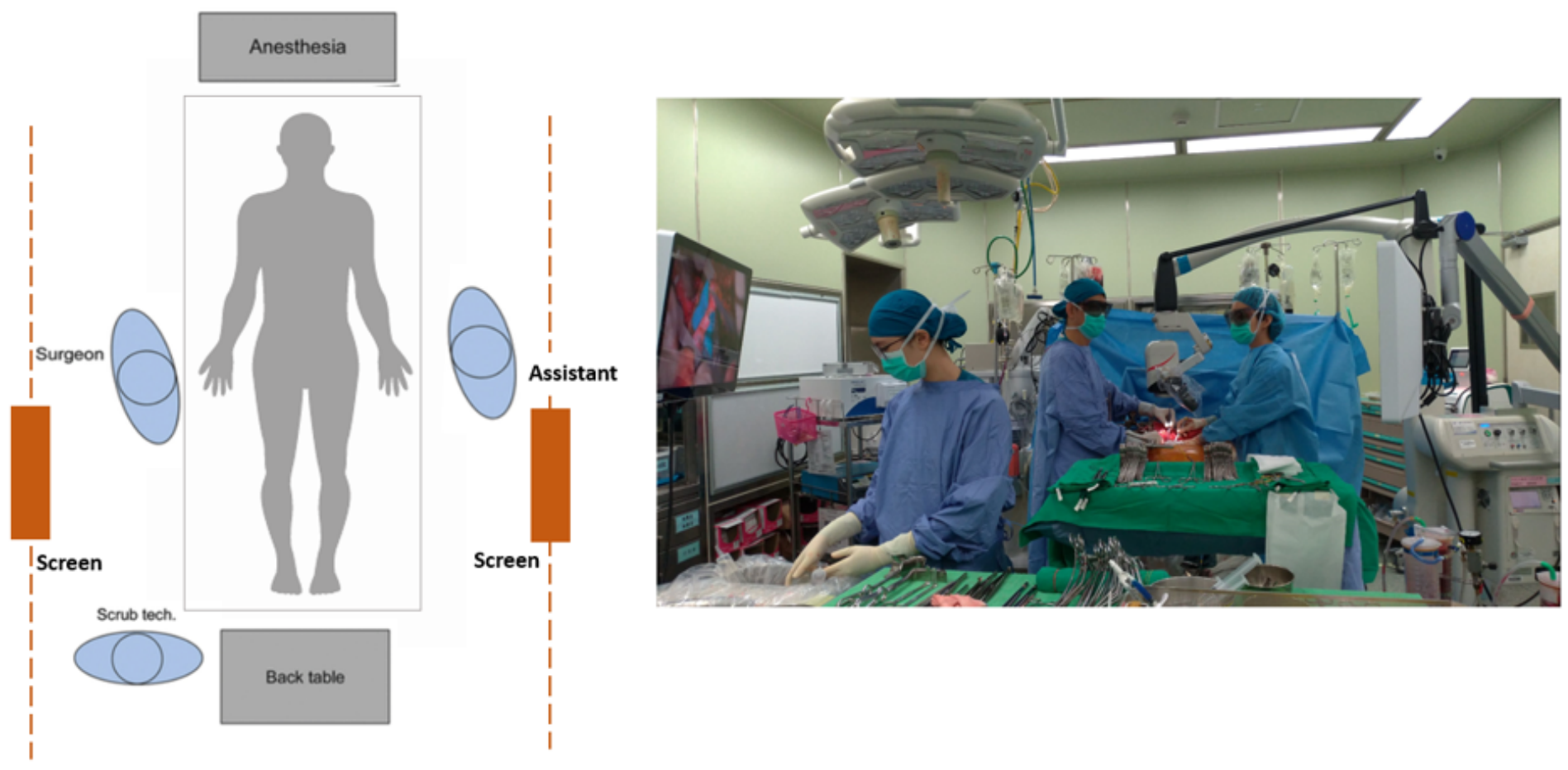

\section{Figure 4}

Operative setup for exoscopic 3D digital microscope.
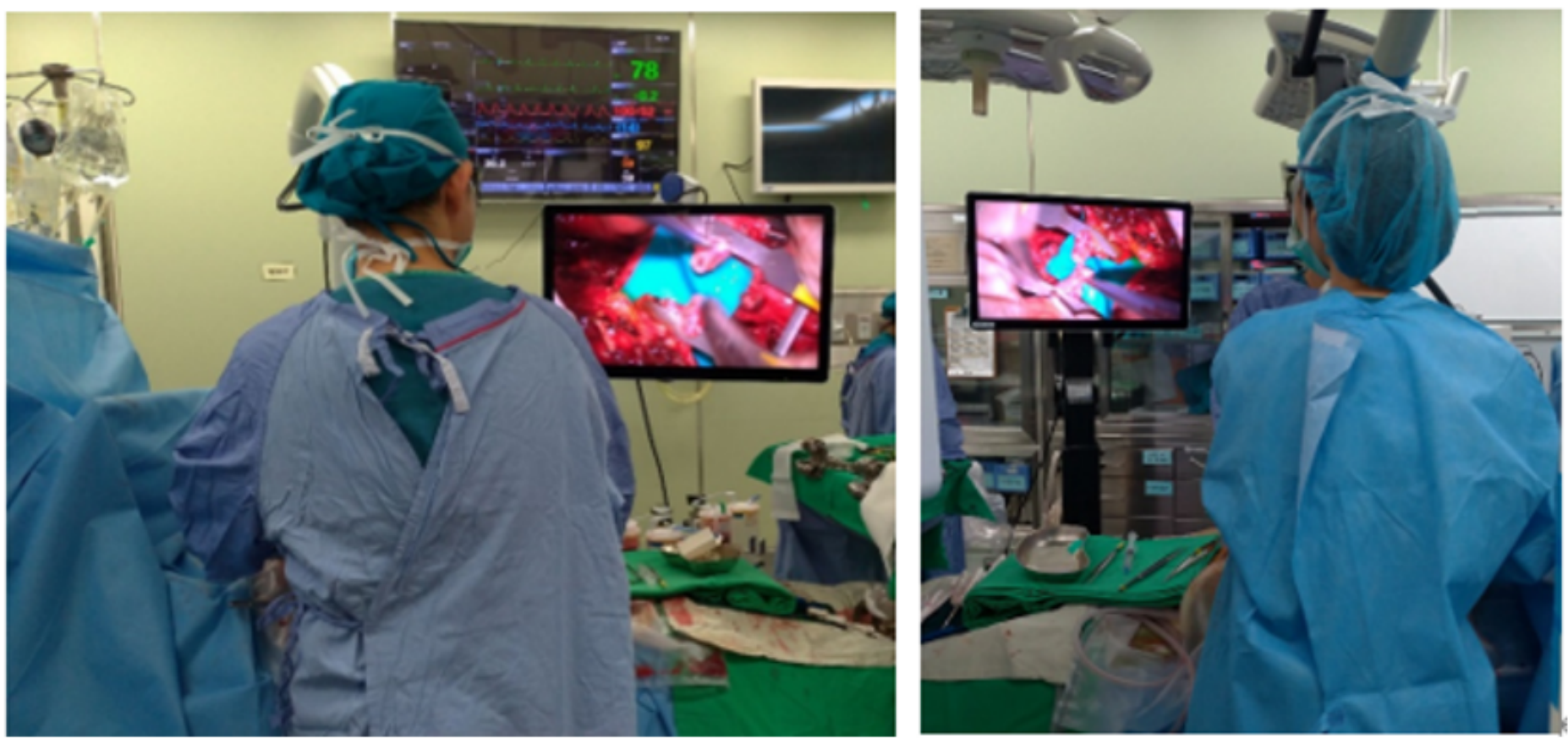

Figure 5

Surgeons operating in an upright position without malposition. 